\title{
Hepatitis C Direct Antiviral Drugs and Hepatic Decompensation in Patients with Advanced Cirrhosis: Culprit or Innocent Bystander?
}

\author{
Varun Saxena $\cdot$ Norah A. Terrault
}

Published online: 14 January 2015

(c) Springer Science+Business Media New York 2015

Interferon-free therapy has revolutionized the treatment of hepatitis $\mathrm{C}(\mathrm{HCV})$ in patients with cirrhosis, including those with decompensated disease. In December 2013, the Food and Drug Administration (FDA) approved simeprevir, a second-generation NS3/4A protease inhibitor, and sofosbuvir, the first-in-class nucleotide analogue NS5B polymerase inhibitor, providing the first opportunity for all oral therapy for HCV. Sofosbuvir with ribavirin was approved for use in patients of all viral genotypes waitlisted for liver transplantation, based upon results from a phase 2 study of patients with Child-Pugh (CP) class A cirrhosis and hepatocellular carcinoma. Simeprevir and sofosbuvir use, initially off-label, was based upon results from the phase 2 COSMOS study [1], where patients with genotype 1 with varying degrees of fibrosis, including compensated cirrhosis, were treated for 12 or 24 weeks. The frequency of sustained virologic response at week 12 (SVR12) reached $93 \%$ (38/41) among those with compensated cirrhosis [1]. Recently, the FDA approved sofosbuvir and simeprevir for treatment of genotype $1 \mathrm{HCV}$, including cirrhotics. However, the safety information for this combination in patients with CP class B cirrhosis is limited.

In this issue of Digestive Diseases and Sciences, Stine et al. [2] describe two patients with CP-B cirrhosis and elevated baseline total bilirubin concentrations (5.3 and $9.5 \mathrm{mg} / \mathrm{dL}$ ) who developed worsening hepatic decompensation during treatment with sofosbuvir and simeprevir. Both patients developed significant elevations of total

V. Saxena $\cdot$ N. A. Terrault $(\bowtie)$

Division of Gastroenterology and Hepatology, University

of California San Francisco, 513 Parnassus Ave, S357,

Box 0538, San Francisco, CA 94143, USA

e-mail: norah.terrault@ucsf.edu bilirubin (indirect fraction unknown), by week 4-5 in Case 1 and by week 2 in Case 2. The authors concluded that simeprevir was "probably" (Case 1) and "possibly" (Case 2) causative of these events, based on the Roussel Uclaf Causality Assessment Method (RUCAM), a system that assigns specific points for clinical, biochemical, serologic, and radiologic features of liver injury to yield a composite score that reflects the likelihood that the hepatic injury is due to a specific medication [3]. Essential aspects of these cases are not included, such as changes in renal function, the results of evaluations for infectious or other causes of acute hepatic decompensation, and if drug-drug interactions may have been contributory. Moreover, the validity of RUCAM criteria in patients with cirrhosis and marked elevations of bilirubin at baseline is questionable. Nonetheless, these two cases highlight the striking changes in clinical status that can occur during HCV treatment for patients with decompensated cirrhosis and the heightened concerns of clinicians regarding use of protease inhibitors in this setting.

Treatment of patients with decompensated cirrhosis, of whom many have renal dysfunction, requires detailed knowledge of antiviral drug metabolism and excretion. Sofosbuvir is extensively metabolized in the liver to the pharmacologically active nucleoside triphosphate analog GS-461203 with eventual dephosphorylation to the inactive metabolite GS-331007 [4]. Relative to subjects with normal hepatic function, the sofosbuvir areas under the curves from 0 to $24 \mathrm{~h}\left(\mathrm{AUC}_{0-24}\right)$ were 130 and $140 \%$ higher in patients with $\mathrm{CP}$ class $\mathrm{B}$ and $\mathrm{C}$ cirrhosis, whereas the GS$331007 \mathrm{AUC}_{0-24}$ was only 18 and $9 \%$ higher, respectively [4]. Further, no dose adjustments for sofosbuvir are recommended for patients with $\mathrm{CP}$ class $\mathrm{B}$ or $\mathrm{C}$ cirrhosis due to the lack of adverse effects with exposure to sofosbuvir and GS-331007 based on population pharmacokinetic 
analysis [4]. Instead, renal clearance is the major elimination pathway for sofosbuvir, via GS-331007. Compared to subjects with normal renal function, the sofosbuvir $\mathrm{AUC}_{0-\infty}$ was 1.7-fold higher and the GS-331007 $\mathrm{AUC}_{0-\infty}$ was 4.5 -fold higher in those with eGFR $<30 \mathrm{~mL} / \mathrm{min} /$ $1.73 \mathrm{~m}^{2}$ [4]. As a result, use of sofosbuvir is not recommended for patients with eGFR $<30 \mathrm{~mL} / \mathrm{min} / 1.73 \mathrm{~m}^{2}$. In contrast, simeprevir is extensively metabolized by the hepatic cytochrome CYP3A system and possibly the CYP2C8 and CYP2C19 systems and eliminated via biliary excretion [5]. Relative to subjects with normal hepatic function, simeprevir $\mathrm{AUC}_{0-24}$ values were 2.4- and 5.2fold higher in patients with $\mathrm{CP}$ class $\mathrm{B}$ and class $\mathrm{C}$ cirrhosis, respectively [5]. In clinical trials, higher exposure to simeprevir was associated with increased frequency of adverse reactions [5]. As a result, it is recommended that risks and benefits be carefully considered prior to simeprevir use in patients with $\mathrm{CP}$ class $\mathrm{B}$ cirrhosis and avoided in patients with $\mathrm{CP}$ class $\mathrm{C}$ cirrhosis [5].

While these pharmacokinetic data are helpful, their utility for predicting adverse drug reactions in the clinic is limited due to lack of data for most drug combinations and for patients with varying degrees of combined liver and renal dysfunction as occurs frequently in patients with $\mathrm{CP}$ class $\mathrm{B}$ and $\mathrm{C}$ cirrhosis. In the absence of more comprehensive data, selecting direct antiviral drugs that are unaffected by liver dysfunction may be a safer option. Certainly, these data highlight the need for careful weighing of risks and benefits of treatment in patients with advanced liver disease, the value of closer monitoring for adverse events in patients with decompensated cirrhosis receiving antiviral therapy and, ideally, the availability of liver transplantation as a rescue therapy should clinical status worsen.

The report by Stine and colleagues highlights the challenge in establishing a causal relationship between drug exposures and decompensating events in patients with advanced cirrhosis. Natural history studies highlight the frequency of decompensating events among patients with cirrhosis (Table 1). Based on data reported for the HALTC study [6], the annualized incidence of decompensation (defined as variceal hemorrhage, ascites, bacterial peritonitis, or encephalopathy) was $12.9 \%$ once patients had developed CP class B (CP score $\geq 7$ ) cirrhosis [6]. The Model of End-stage Liver Disease (MELD), which predicts 3-month mortality, indicates that the two cases reported by Stine et al. with MELD scores of 17 and 20 had an estimated 3-month mortality risk of $6.0 \%$ and $19.6 \%$, respectively [7]. Recognizing that decompensating events can occur independently of treatment exposure, there is a need for controlled studies to ascertain which direct antiviral drugs increase the risk of decompensation.

In the absence of controlled studies, the substantial "real life" experience with sofosbuvir and simeprevir is informative
Table 1 Annualized incidence of each clinical outcome following CP score $\geq 7$

\begin{tabular}{lc}
\hline Outcome & $\begin{array}{l}\text { Events after CP } \\
\text { score } \geq 7 \\
(n=137)(\%)\end{array}$ \\
\hline Variceal hemorrhage & 1.2 \\
Ascites & 12.7 \\
Encephalopathy & 10.3 \\
HCC or presumed HCC & 4.5 \\
All-cause death & 10.0 \\
All-cause death or liver transplantation & 14.7 \\
Liver-related death & 8.7 \\
Liver-related death or liver transplantation & 14.3 \\
Decompensation (variceal hemorrhage, ascites, & 12.9 \\
$\quad$ bacterial peritonitis, encephalopathy) & 14.8 \\
HCC/presumed HCC or decompensation & \\
$\quad$ (variceal hemorrhage, ascites, bacterial & \\
peritonitis, encephalopathy) & \\
\hline Adted fipt Table of Dienstag et &
\end{tabular}

Adapted from Table 2 of Dienstag et al.'s [6] HALT-C trial

(Table 2). The HCV-TARGET cohort of 2,063 patients treated with sofosbuvir-including regimens included 1,126 treated with sofosbuvir and simeprevir (243 treated with ribavirin), of which 577 had cirrhosis and 227 with history of decompensation [8]. Serious adverse events were similar in patients treated with sofosbuvir plus ribavirin $(7.9 \%)$ versus sofosbuvir plus simeprevir (4.6 and $7.5 \%$ if ribavirin added) [8]. Twelve deaths were reported, the majority ( 9 of $12,75 \%$ ) in patients with cirrhosis; eight among patients treated with simeprevir and sofosbuvir with and without ribavirin (8 of $1,126,0.7 \%$ ) [8]. In composite, available preliminary data suggest that simeprevir and sofosbuvir can be safely administered in those with mildly decompensated disease with a low rate of adverse events, although serious events including deaths occur.

For patients with decompensated cirrhosis who are not candidates for transplantation, antiviral therapy may be their only hope for survival. For patients with decompensated cirrhosis on the transplantation waiting list, the benefit of antiviral therapy includes prevention of posttransplant recurrence and possibly reversal of decompensation [11]. Certainly, drawing upon the experience of antiviral therapy in $\mathrm{HBV}$-infected patients with decompensated disease suggests that avoiding the need for transplant should be an attainable goal in many HCV patients if viral eradication is achieved [12]. Nonetheless, in contrast to the HBV treatment experience, the HCV drug armamentarium includes multiple drug classes, multiple drug combinations, and higher risk of adverse effects. The best outcome of therapy would be reversal of decompensation and avoidance of need for transplantation. The worse outcome would be worsening of decompensation to the point that transplantation is no longer an option. If drug 
therapy substantially increases the risk of decompensating events, either via drug toxicity or related to viral clearance, then deferring treatment until post-transplant may be the preferred strategy. Since safe and highly effective therapies are available for transplant recipients, treatment may be easier post-transplant when liver function is restored and renal function stable [11].

Overall, implicating a drug as a cause of hepatic decompensation requires a substantial amount of data [3]. The cases reported by Stine et al. serve to raise awareness but are insufficient to meet that burden of proof. Among patients with decompensated cirrhosis whose natural history is prone to further decompensation [6], rigor is required to separate the effects of drug versus natural history, likely best accomplished with the use of matched, untreated control groups within the context of large cohorts. Moving forward, as additional new HCV combinations are approved for treatment of patients with cirrhosis, continued scrutiny of the frequency of adverse events within the population with decompensated cirrhosis combined with robust assessment of the cause of decompensation will further improve therapy of $\mathrm{HCV}$-infected patients complicated by cirrhosis.

\section{References}

1. Lawitz E, Sulkowski MS, Ghalib R, et al. Simeprevir plus sofosbuvir, with or without ribavirin, to treat chronic infection with hepatitis $\mathrm{C}$ virus genotype 1 in non-responders to pegylated interferon and ribavirin and treatment-naive patients: the COSMOS randomised study. Lancet. 2014;384:1756-1765.

2. Stine JG, Intagliata N, Shah NL, et al. Hepatic decompensation likely attributable to simeprevir in patients with advanced cirrhosis. Dig Dis Sci. (Epub ahead of print). doi:10.1007/s10620014-3422-x

3. Danan G, Benichou C. Causality assessment of adverse reactions to drugs-I. A novel method based on the conclusions of international consensus meetings: application to drug-induced liver injuries. J Clin Epidemiol. 1993;46:1323-1330.

4. Sofosbuvir [package insert]. Foster City, CA: Gilead Sciences; 2013.

5. Simeprevir [package insert]. Titusville, NJ: Janssen Therapeutics; 2013.

6. Dienstag JL, Ghany MG, Morgan TR, et al. A prospective study of the rate of progression in compensated, histologically advanced chronic hepatitis C. Hepatology. 2011;54:396-405.

7. Kamath PS, Kim WR. Advanced Liver Disease Study G. The model for end-stage liver disease (MELD). Hepatology. 2007; 45:797-805.

8. Jensen DM, O'Leary JG, Pockros PJ, et al. Safety and efficacy of sofosbuvir-containing regimens for hepatitis $\mathrm{C}$ : real-world experience in a diverse, longitudinal observational cohort [abstract]. In: Program and Abstracts of the 65th Annual Meeting of the American Association for the Study of Liver Diseases, 7-11 November 2014, Boston, MA, Abstract 45.

9. Aqel B, Pungpapong S, Werner TK, et al. The use of simeprevir and sofosbuvir to treat $\mathrm{HCV}$ G1 in the liver transplant setting: 
The experience in 3 US Centers [abstract]. In: American Association for the Study of Liver Diseases International Conference, 7-11 November 2014, Boston, MA, Abstract 19.

10. Modi AA, Nazario H, Gonzalez SA, et al. Safety and efficacy of simeprevir + sofosobuvir with or without ribavirin in patients with decompensated genotype 1 hepatitis $\mathrm{C}$ induced cirrhosis [abstract]. In: American Association for the Study of Liver
Diseases International Conference, 7-11 November 2014, Boston, MA, Abstract 956.

11. Price J, Terrault NA. Treatment of hepatitis C in liver transplant patients. Liver Transplantation. 2015 (in press).

12. Schiff ER, Lai CL, Hadziyannis S, et al. Adefovir dipivoxil therapy for lamivudine-resistant hepatitis B in pre- and post-liver transplantation patients. Hepatology. 2003;38:1419-1427. 